\title{
Myelography CPT Coding Updates: Effects of 4 New Codes and Unintended Consequences
}

\author{
(D)F.H. Chokshi, (DR.K. Tu, (D) G.N. Nicola, and (D).A. Hirsch
}

\begin{abstract}
SUMMARY: The Current Procedural Terminology of the American Medical Association has recently introduced coding changes for myelography with the introduction of new bundled codes. The aim of this review was to help neuroradiologists understand these code changes and their unintended consequences and to discuss various scenarios in which permutations of various codes could occur in clinical practice.
\end{abstract}

ABBREVIATIONS: $\mathrm{AMA}=$ American Medical Association; $\mathrm{CPT}=$ Current Procedural Terminology

$\mathbf{T}$ he Current Procedural Terminology (CPT) system has been the national medical coding standard in the United States ${ }^{1}$ since 2000. CPT, owned and trademarked by the American Medical Association (AMA), provides physicians, including neuroradiologists, an opportunity to directly participate in the creation of procedural codes. The work of the CPT Editorial Panel and advisors is published in the CPT manual and educational materials in an effort to provide coding clarity and preserve the intended coding integrity of medical procedures. The effects of this recent bundling, ${ }^{2,3}$ including the interplay among radiologic modalities, are important for neuroradiologists. The authors illustrate these complexities with recent updates to the differentiation of $\mathrm{x}$-ray and CT with intrathecal contrast media and the unintended consequences of revaluation of the original codes. This review will provide clarity for physicians and coders who wish to enhance their familiarity with these changes. Of note, we intentionally

Received November 17, 2015; accepted November 20.

From the Division of Neuroradiology (F.H.C.), Department of Radiology and Imaging Sciences, Emory University School of Medicine, Atlanta, Georgia; Department of Radiology (R.K.T.), Progressive Radiology, NFPHC/United Medical Center, Washington, DC; Department of Radiology (R.K.T.), BridgePoint Hospital Hadley, BridgePoint Subacute and Rehabilitation Hadley, BridgePoint Hospital Capitol Hill, Washington, DC; Department of Radiology (R.K.T.), George Washington University, Washington, DC; Hackensack Radiology Group (G.N.N.), Hackensack University Medical Center, River Edge, New Jersey; and Neuroendovascular Division (J.A.H.), Massachusetts General Hospital, Harvard Medical School, Boston, Massachusetts.

Falgun H. Chokshi, MD, MS, is an Association of University Radiologists GE Radiology Research Academic Fellow (2015-2017). This investigation was supported, in part, by the Association of University Radiologists GE Radiology Research Academic Fellowship Award.

Please address correspondence to Falgun H. Chokshi, MD, MS, Division of Neuroradiology, Department of Radiology and Imaging Sciences, Emory University School of Medicine, 1364 Clifton Rd NE, Atlanta, GA 30068; e-mail: falgun.chokshi@ emory.edu; @FalgunChokshiMD

http://dx.doi.org/10.3174/ajnr.A4666 used "providers" instead of "physicians" because allied health professionals or other qualified health care providers (eg, nurse practitioners) can also perform and increasingly code these procedures, ${ }^{4,5}$ especially because diagnostic imaging volume has increased with time. ${ }^{6}$

\section{Definition of Myelography}

Myelography is a comprehensive radiographic examination of the spine following intrathecal injection of iodinated contrast media, involving assessment of static structures such as the spinal canal and each exit foramen, and dynamic, real-time assessment of contrast injection and its flow dynamics under direct visualization. ${ }^{7}$ Fluoroscopic gastrointestinal studies require maneuvers to move contrast; so too are patient and table maneuvers often used as tools to evaluate canal stenosis, leaks, complications of surgery, and spinal instrumentation. A myelogram is not a single image documenting needle placement but a comprehensive evaluation of the contrast-enhanced thecal sac with assessment of each exit foramen for pathology. Specialized techniques such as flexion, rotation, and hyperextension to show spinal stenosis and lateral recess stenosis are tools chosen by the provider as trouble-shooting techniques of assessment as contrast disperses in the thecal sac. Perhaps, the single most important message of this article is that one should not code for a myelogram when one is using $\mathrm{x}$-ray imaging simply to document the intrathecal location of the needle placement and contrast injection.

\section{Bundling of Myelography CPT Codes}

The CPT 2015 Professional Edition ${ }^{8}$ introduced 4 new bundled codes, increasing the total number to 8 . The new codes combined intrathecal contrast administration via lumbar injection, fluoroscopic guidance, and $\mathrm{x}$-ray myelographic radiologic supervision 
Table 1: CPT codes for myelography

\begin{tabular}{lcccc}
\hline & Nonbundled Code & & Bundled Code \\
\cline { 2 - 3 } \multicolumn{1}{c}{ Myelographic Region } & $\begin{array}{c}\text { (2 Separate Providers: 1 Doing } \\
\text { Injection and Other Doing } \\
\text { Myelogram Interpretation) }\end{array}$ & & $\begin{array}{c}\text { (1 Provider Doing Both } \\
\text { Injection and Myelogram } \\
\text { Interpretation) }\end{array}$ \\
\hline Cervical & 72240 & 62302 \\
Thoracic & 72255 & 62303 \\
Lumbar & 72265 & 62304 \\
\pm 2 Regions & 72270 & 62305 \\
Intrathecal injection only and & $62284+77003^{\text {a }}$ & & Not Applicable \\
fluoroscopic guidance code & & & \\
\hline
\end{tabular}

a No myelography at all; injection and CT spine with contrast only.

Table 2: CPT codes for CT spine with contrast ${ }^{a}$

\begin{tabular}{lc}
\hline Spine Level & CPT Code \\
\hline Cervical & 72126 \\
Thoracic & 72129 \\
Lumbar & 72132 \\
\hline
\end{tabular}

add Modifier 59 if x-ray myelography is performed on the same day on the same patient by the same provider.

\section{Intrathecal Injection}
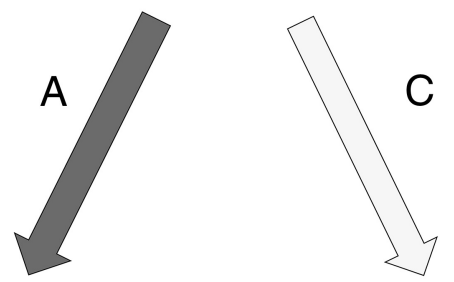

\section{Myelogram

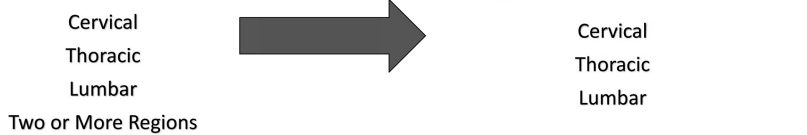

FIG 1. Permutations of myelography coding. A, B, and C denote paths of coding.

and interpretation into codes used only when the same provider performs both injection and myelography on the same day on the same patient. Bundling occurred following recommendations by the Relativity Assessment workgroup screen. The Relativity Assessment workgroup, one of many committees of the Relative Value Scale Update Committee of the AMA, screens for procedures occurring together $75 \%$ of the time. Existing codes were not replaced because there are scenarios in which one provider injects the lumbar spine with contrast media and another provider interprets the study. ${ }^{2,3}$ Table 1 shows both nonbundled and bundled CPT codes for myelography.

\section{Modifier 59}

Distinct from the Relativity Assessment workgroup screen of the AMA, the National Correct Coding Initiative of the Centers for Medicaid and Medicare Services reviews codes for potential misuse. The National Correct Coding Initiative flagged myelography and contrast-enhanced CT of the spine and submitted a query to the AMA requesting clarification. Because myelography and contrast-enhanced CT of the lumbar spine occurred in the same patients, the National Correct Coding Initiative was concerned that these concurrent procedures may be unnecessary and duplicative. Subspecialty societies including the American Society of Neuroradiology replied and provided detailed explanations clarifying myelography and contrast-enhanced CT as distinct procedures. Accepting the explanation, the National Correct Coding Initiative recommended that Modifier 59, Distinct Procedural Service, be added to CPT codes if the CT examination is performed following myelography on the same patient on the same day for the reason described above. Table 2 provides CPT codes for CT of the spine with contrast, which have been used since July 1, 2014. Modifier 59 flags and clarifies procedures that may be mistaken as duplicative. Again, this modifier is not applied if $\mathrm{x}$-ray images were merely obtained to check intrathecal spinal needle placement (code 62284). Only in this latter situation can code 62284 be combined with billing code 77003 , the code for fluoroscopic guidance.

\section{Coding Permutations for Myelography}

To help radiologists and coders understand these coding changes further, we have included a set of scenarios to show various permutations of myelography coding.

Scenario 1 involves intrathecal lumbar injection only (code 62284) followed by CT of the spine with contrast (codes 7212673132) (Fig 1, path C). This scenario does not include $\mathrm{x}$-ray myelography at all; therefore, neither bundled codes nor Modifier 59 is used. Scenario 1 can include a single provider injecting and interpreting the CT or a single provider injecting (eg, nurse practitioner) and another provider interpreting the CT (eg, neuroradiologist). Again, scenario 1 also allows billing of code 77003, a separate code for fluoroscopic guidance. Code 77003 can only be billed with 62284 if none of the new bundled codes are used (62302-62305), which already account for fluoroscopic guidance. For example, a patient undergoing an intrathecal lumbar injection only followed by CT lumbar spine with contrast would be coded as $62284+77003+72132$. For C1-C2 injection only, use code 61055 instead of 62284 .

Scenario 2 would include both x-ray myelography and CT spine interpretation by a single provider performing both parts (Fig 1, paths A and B). Most important, this scenario warrants the new bundled myelography codes in the last column of Table 1 and the CT spine codes in Table 2 plus Modifier 59. In this scenario, do not bill for fluoroscopic guidance, code 77003 . Therefore, a patient receiving intrathecal lumbar injection + lumbar $\mathrm{x}$-ray myelography + CT lumbar spine with contrast would be coded as 62304 (bundled) $+72132+$ Modifier 59.

Scenario 3, albeit presumably rare, would involve one provider doing the intrathecal lumbar injection and a second provider performing myelography and interpreting the $\mathrm{CT}$ of the spine with contrast (Fig 1, paths A and B). In this scenario, provider 1 performs the intrathecal lumbar injection, which is coded as $62284+77003$. Provider 2 performs the $\mathrm{x}$-ray myelography, which is coded with an old (unbundled) $x$-ray myelography code 
(72240-72270). In addition, provider 2 interprets the CT spine with contrast, which is coded with a CT with contrast code (7212672132) plus Modifier 59. If no myelography is performed, then provider 1 performs intrathecal injection only $(62284+77003)$ and provider 2 interprets the CT spine with contrast, which is coded 72126-73132 without Modifier 59 (scenario 1).

\section{CONCLUSIONS}

Myelography is a procedure that predates cross-sectional imaging and provides unique radiographic information as dynamic, realtime imaging. These studies are commonly obtained and interpreted by neuroradiologists. During the past several years, as other imaging modalities became available to evaluate the spine, interest in these modalities by the AMA and Centers for Medicaid and Medicare Services increased. The screens ${ }^{9}$ of the AMA for codes reported together above a certain predefined threshold were bundled as a result of being identified by the Relativity Assessment Workgroup, thereby adding 8 codes for myelography. The Centers for Medicaid and Medicare Services introduced Modifier 59 for CT. In addition to new codes, there were new valuations of the bundled and pre-existing nonbundled codes, an unintended consequence. It is very important that neuroradiologists and their coders understand the nuances of myelography coding based on changes in 2015 to maintain coding integrity of the procedures that are performed and to avoid Recovery Audit Contractor audits. Proper coding ensures appropriate reimbursement and accurate retrospective use of physician work.

\section{ACKNOWLEDGMENTS}

The authors acknowledge Rahul Bhala, MBA, MPH (Managing Director-Economics and Health Policy, American Society of Neuroradiology), and Jacqueline A. Bello, MD, FACR (Professor of Clinical Radiology and Neurosurgery, Director of Neuroradi- ology, Albert Einstein College of Medicine, Montefiore Medical Center), for their assistance.

Disclosures: Joshua A. Hirsch—UNRELATED: Consultancy: Medtronic (ongoing, related to the spine), CareFusion (taught single course in last 36 months, not ongoing).

\section{REFERENCES}

1. Hirsch JA, Leslie-Mazwi TM, Nicola GN, et al. Current procedural terminology; a primer. J Neurointerv Surg 2015;7:309-12 CrossRef Medline

2. Hirsch JA, Donovan WD, Leslie-Mazwi TM, et al. Component coding and the neurointerventionalist: a tale with an end. J Neurointerv Surg 2013;5:615-19 CrossRef Medline

3. Hirsch JA, Silva E 3rd, Nicola GN, et al. The RUC: a primer for neurointerventionalists. J Neurointerv Surg 2014;6:61-64 CrossRef Medline

4. Hawkins CM, Bowen MA, Gilliland CA, et al. The impact of nonphysician providers on diagnostic and interventional radiology practices: operational and educational implications. J Am Coll $\mathrm{Ra}$ diol 2015;12:898-904 CrossRef Medline

5. Chittle MD, Vanderboom T, Borsody-Lotti J, et al. An overview of clinical associate roles in the neurointerventional specialty. J Neurointerv Surg 2015 Jan 5. [Epub ahead of print] CrossRef Medline

6. Chokshi FH, Hughes DR, Wang JM, et al. Diagnostic radiology resident and fellow workloads: a 12-year longitudinal trend analysis using national Medicare aggregate claims data. J Am Coll Radiol 2015;12:664-69 CrossRef Medline

7. Myelography. RadiologyInfo. Org; 2015. http://www.radiologyinfo. org/en/info.cfm?pg=myelography. Accessed November 11, 2015

8. CPT 2015 Professional Edition. American Medical Association; 2015. https://commerce.ama-assn.org/store/catalog/productDetail.jsp? product_id $=$ prod $2480008 \&$ navAction $=$ push. Accessed November 11,2015

9. Donovan WD, Leslie-Mazwi TM, Silva E 3rd, et al. Diagnostic carotid and cerebral angiography: a historical summary of the evolving changes in coding and reimbursement in a complex procedure family. J Neurointerv Surg 2014;6:712-17 CrossRef Medline 